\title{
A Givental-like formula and bilinear identities for tensor models
}

\section{Stéphane Dartois}

LIPN, Institut Galilée, CNRS UMR 7030, Université Paris 13, F-93430, Villetaneuse, France

Laboratoire de Physique Théorique, CNRS UMR 8627, Université Paris 11, 91405 Orsay Cedex, France

E-mail: stephane.dartois@outlook.com

ABSTRACT: In this paper we express some simple random tensor models in a Giventallike fashion i.e. as differential operators acting on a product of generic 1-Hermitian matrix models. Finally we derive Hirota's equations for these tensor models. Our decomposition is a first step towards integrability of such models.

KEYwords: Matrix Models, Integrable Equations in Physics, Models of Quantum Gravity

ARXIV EPRINT: 1409.5621 


\section{Contents}

1 Introduction 1

2 Matrix models 3

2.1 Generalities on matrix models 3

2.2 Generic matrix model and Kontsevitch model 5

3 Tensor models $\quad 6$

3.1 Tensor invariants and generic 1-tensor model $\quad 6$

$\begin{array}{lll}3.2 & T^{4} \text { tensor models and intermediate field representation } & 9\end{array}$

4 Constructing $T^{4}$ tensor model out of matrix models 10

5 Bilinear identities for $T^{4}$ tensor model $\quad 13$

$\begin{array}{lll}6 & \text { Conclusion } & 15\end{array}$

\section{Introduction}

Tensor models are the natural generalization of matrix models. Initially introduced in the context of nuclear physics, random matrices have been applied extensively from statistical physics to number theory. A major development was the introduction of interacting random matrix models and of their $1 / N$ expansion. Indeed it was discovered that the Feynman graphs of these models implement consistently a sum over cellular decompositions of Riemann surfaces naturally encoding the topology of the discretized surfaces [23]. This feature made them central both to string theory and to two dimensional quantum gravity. Indeed the double scaling limit of matrix models provides a road towards the still ongoing non-perturbative definition of string theory. Moreover the relationship between the continuum Liouville formulation and discretized matrix models plays an ever increasing role for the understanding of two-dimensional quantum gravity.

Early tensor models were introduced $[1,2]$ in order to generalize to higher dimensions this great success of matrix models. Unfortunately they turn out to be difficult to handle analytically. Some key concepts of matrices (eigenvalues, characteristic polynomials, determinants) simply do not generalize (in a easily computable way) to higher rank tensors. Moreover the much richer geometries in higher dimensions obviously bring new challenges. Which description of geometry is most naturally and in the simplest way associated to a Feynman theory? This question, which was certainly not central from a purely mathematical point of view, is the crucial one for a field theoretic-type quantization of gravity in higher dimensions. Graph Encoded Manifolds (GEM) theory and crystallization [30, 31] 
in fact turned out to provide an answer to that question. Indeed colored triangulations are dual to simple field theoretic combinatorics.

Following this observation, colored tensor models were introduced [26]. Unexpectedly they also solve many associated difficulties. First and foremost they allowed to find a tensor analog of the matrix $1 / N$ expansion [32].

A major new feature of this expansion is that it is not of topological nature (at least not in a naive way). The full meaning of the parameter governing this expansion, called the degree, is still unclear, although it can be computed rather easily as the sum of the genera of normal surfaces embedded in the cellular decomposition of the discretized geometry [33]. Progress followed quickly, in particular through computation of single [34, 44] and double scaling limits of such models [9-11], and the inclusion of matter in the corresponding random geometry [38, 39].

From the start tensor models were also related to the group field theory (GFT) approach to quantum gravity $[21,41,43]$. This approach implements a sum over spin networks of loop quantum gravity [22] as a quantum field theory defined on a Lie group. Tensor models provided GFT with a consistent class of interactions and observables which generalize the concept of locality to a background-independent formulation. This improvement allowed to renormalize GFT [36, 42]. A complete study has been achieved in [37], interesting physical perspectives are enumerated in [25].

Over the years, matrix models have developed a lot of additional exciting features. First they could be used to tackle combinatorial problems, such as counting a variety of 2-dimensional maps [12]. Using a new class of matrix models, Kontsevich proved Witten's conjecture on generating functions for intersection numbers of moduli spaces. Matrix models also have rich integrability properties, unraveled through orthogonal polynomials, KdV hierarchy and Hirota's equations. More recently Givental introduced a decomposition of solutions to the multi-components KP hierarchy using matrix models [14, 15]. An other important development which aroses from matrix models around the same time is the topological recursion [16], which has grown into a polyvalent technique allowing to solve many problems of algebraic and enumerative geometry [17]. The relation between these two techniques has been shown in [19].

It is then natural to ask whether tensor models inherit, at least partly, of these important mathematical features. In this paper we derive a decomposition formula that looks like (but is not) a Givental decomposition formula for the simplest random tensor model, namely the quartic melonic model (at any rank). In fact we describe the partition function of this model as the action of a differential operator on a product of Hermitian 1-matrix models (the number of matrix models being given by the rank of the tensor models). We end this paper by deriving bilinear identities for the tensor model by deforming the Hirota's equations satisfied by the Hermitian 1-matrix model.

This paper is structured as follow:

- Section 2 about matrix models recalls the well known results on the subject.

- Section 3 introduces tensor models more formally, then specializes to the quartic melonic tensor model and derives the intermediate field representation for it. 
- Section 4 shows the decomposition formula for tensor model into Hermitian matrix models.

- Section 5 recalls the basic Hirota's equations for matrix models and derives bilinear identities for the quartic melonic tensor model from them.

\section{Matrix models}

\subsection{Generalities on matrix models}

For pedagogical reasons I present some known results about matrix models in this section, this should allow readers coming from different communities to read this paper easily. Most of the material can be found in $[3,4]$.

Matrix $1 / N$ development. We recall briefly the $1 / N$ development of matrix models. Consider the matrix model defined by

$$
Z\left[t_{4}, N\right]=\int d M \exp \left(-N\left(\frac{1}{2} \operatorname{Tr}\left(M^{2}\right)+\frac{t_{4}}{4} \operatorname{Tr}\left(M^{4}\right)\right)\right)
$$

with $N$ the size of the matrix. At the formal level this is a generating function for quadrangulations. The free energy $F=\ln Z$ expands as $F=\sum_{g \geq 0} N^{2-2 g} F_{g}\left(t_{4}\right)$ where the $F_{g}$ 's are generating functions of quadrangulations of genus $g$ for the counting variable of quadrangles $t_{4}$. In the limit $N \rightarrow \infty$ only the leading order survives i.e. the term $F_{0}$ counting the planar quadrangulations, so to say quadrangulations of the sphere $S^{2}$. One can compute the two points function $G_{2}\left(t_{4}\right)=\frac{1}{N}\left\langle\operatorname{Tr}\left(M^{2}\right)\right\rangle$ in this limit and recover the Tutte's result [24] for planar rooted quadrangulations:

$$
G_{2}\left(t_{4}, N=\infty\right)=\sum_{n} 2 \frac{3^{n}}{(n+2)(n+1)}\left(\begin{array}{c}
2 n \\
n
\end{array}\right) t_{4}^{n} .
$$

Matrix double scaling limit. One expands the free energy in $N$ :

$$
F\left(t_{4}, N\right)=\sum_{g \geq 0} N^{2-2 g} F_{g}\left(t_{4}\right)
$$

All $F_{g}$ 's have a critical point at $t_{4}=t_{c}$. Roughly they behave as $F_{g} \sim C_{g}\left(t_{4}-t_{c}\right)^{\frac{5}{4}(2-2 g)}$. By setting $x=$ constant $=N\left(t_{4}-t_{c}\right)^{5 / 4}$ while $N \rightarrow \infty$ and $t_{4} \rightarrow t_{c}$ one gets the double scaling limit of $F$ :

$$
F(x)=\sum_{g \geq 0} x^{2-2 g} C_{g}
$$

The $C_{g}$ coincide with the correlations functions of Liouville gravity. This corresponds to the continuum limit of matrix models. One can thus understand that $F(x)$ should satisfy some differential equations, that is, the differential equation satisfied by the Liouville partition function. This equation is of the Painlevé type. 
Orthogonal polynomials and integrability. This presentation is based on $[8,12]$. Orthogonal polynomials allows to compute exactly the partition function of matrix model. For the sake of definitness consider the matrix model defined above with potential $V(M)=$ $\frac{t_{4}}{4} \operatorname{Tr} M^{4}$. The orthogonal polynomials are the only polynomials which are monic and orthogonal with respect to the measure $\exp \left(-\frac{t_{4}}{4} x^{4}\right) d x$ :

$$
\int P_{N, t_{4}}(x) P_{M, t_{4}}(x) \exp \left(-N \frac{t_{4}}{4} x^{4}\right) d x=\delta_{N, M} K_{N},
$$

$K_{N}$ a proportionality factor. This can be used to solve matrix models because the orthogonality relations determine completely the model. For instance an orthogonal polynomial is provided by $P_{N, t_{4}}(x)=\langle\operatorname{det}(x-M)\rangle$. Following [12], changing variables to eigenvalues leads to:

$$
\begin{aligned}
P_{N, t_{4}}(x) & =\langle\operatorname{det}(x-M)\rangle \\
& =\frac{1}{Z_{N}} \int \prod_{i=1}^{N} d \mu\left(x_{i}\right)\left(x-x_{i}\right) \prod_{i<j}\left(x_{i}-x_{j}\right)^{2},
\end{aligned}
$$

where $d \mu(x)=\exp (-N V(x)) d x$. Then write:

$$
\begin{aligned}
Z_{N} & \int d \mu(x) P_{N, t_{4}}(x) x^{M} \\
& =\int d \mu(x) \int \prod_{i=1}^{N} d \mu\left(x_{i}\right)\left(x-x_{i}\right) \Delta\left(\left\{x_{j}\right\}\right)^{2} x^{M} \\
& =\int \prod_{i=1}^{N+1} d \mu\left(x_{i}\right) \Delta\left(\left\{x_{j}\right\}_{j=1 \cdots N+1}\right) \Delta\left(\left\{x_{j}\right\}_{j=1 \ldots N}\right) x_{N+1}^{M} \\
& =\frac{1}{N+1} \sum_{k}(-1)^{N+1-k} \int \prod_{i=1}^{N+1} d \mu\left(x_{i}\right) \Delta\left(\left\{x_{j}\right\}_{j=1 \cdots N+1}\right) \Delta\left(x_{1}, \cdots, \hat{x_{k}}, \cdots, x_{N+1}\right) x_{k}^{M} .
\end{aligned}
$$

Noticing that:

$$
\frac{1}{N+1} \sum_{k}(-1)^{N+1-k} \Delta\left(x_{1}, \cdots, \hat{x_{k}}, \cdots, x_{N+1}\right) x_{k}^{M}=\left|\begin{array}{ccccc}
1 & x_{1} & \cdots & x_{1}^{N-1} & x_{1}^{M} \\
1 & x_{2} & \cdots & x_{2}^{N-1} & x_{2}^{M} \\
\vdots & \cdots & \cdots & \cdots & \vdots \\
1 & x_{N+1} & \cdots & x_{N+1}^{N-1} & x_{N+1}^{M}
\end{array}\right|,
$$

this determinant vanishes for $M \leq N-1$. For $M=N$ :

$$
Z_{N} \int d \mu(x) P_{N, t_{4}}(x) x^{N}=\frac{1}{N+1} \int \prod_{i=1}^{N+1} d \mu\left(x_{i}\right) \Delta\left(\left\{x_{j}\right\}_{j=1 \cdots N+1}\right)^{2}=\frac{Z_{N+1}}{N+1} .
$$

This implies $K_{N}=\frac{Z_{N+1}}{(N+1) Z_{N}}$. So one computes $K_{N}$ as a function of the $Z_{N}$ 's:

$$
K_{N}=\frac{Z_{N+1}\left[t_{4}\right]}{(N+1) Z_{N}\left[t_{4}\right]} .
$$

Finally this leads to $Z_{N}\left[t_{4}\right]=N ! \prod_{i=1}^{N-1} K_{i}$. One then derives recursion relations for the $K_{N}$ (for instance see [8] for a general description of these problems). 


\subsection{Generic matrix model and Kontsevitch model}

In this section we introduce the generic matrix model.

Definition 1. We define the Hermitian one matrix model by the partition function:

$$
Z_{1 M M}\left[\left\{t_{p}\right\}_{p=0 \ldots \infty}, N\right]=\int_{H_{N}} d M \exp \left(-\frac{N}{2} \operatorname{Tr}\left(M^{2}\right)-N \sum_{p \geq 0} t_{p} \operatorname{Tr}\left(M^{p}\right)\right),
$$

$H_{N}$ being the space of $N \times N$ hermitian matrices. This partition function has to be understood at the formal level.

The second term entering the definition of the generic matrix model is called the generic potential, each term of its development is an invariant of the matrix $M$ in such a way that the "action" is univalued. For these invariants to be independent one has to take the limit of big size $N$ of the matrix. Each invariant can be labelled by an integer $p$, and we introduce one coupling constant for each of these invariants. We consider these coupling constants as formal parameters of a formal series obtained by expanding the exponential and interchanging the order of summation. The integral representation introduced above is just a reminder for writing the term of the corresponding formal series (although this integral is well defined for negative values of the coupling constant).

The Kontsevitch model is the model computing the intersection numbers of moduli spaces of Riemann surfaces of genus $g$ and $n$ punctures.

Definition 2. The Kontsevitch model is here defined by:

$$
Z_{K}[\Lambda]=\int_{H_{N}} d X \exp \left(-\operatorname{Tr}(X \Lambda X)+i \operatorname{Tr}\left(X^{3}\right)\right)
$$

where $\Lambda$ is a diagonal matrix. We call the Miwa's coordinates the $T_{k}=\frac{1}{k} \operatorname{Tr}\left(\Lambda^{k}\right)$.

These two models are important since they are used to decompose solutions of the multi-component KP hierarchy as an intertwining operator acting on a product of the matrix models described above i.e. prototypically:

$$
\mathcal{Z}=e^{\mathcal{U}} \prod_{k} Z_{1 M M}\left[\left\{t_{p}^{k}\right\}\right]=e^{\hat{\mathcal{U}}} \prod_{k} Z_{K}\left[\Lambda_{k}\right]
$$

where $\mathcal{Z}$ is given from a spectral curve $S(x, y)$ by $\mathcal{Z}=e^{-F(S)} . F(S):=\sum_{g \geq 0} N^{2-2 g} F_{g}(S)$ is the generating function of genus $g$ symplectic invariants ${ }^{1}$ (the $F_{g}$ 's) of $S$. The intertwining operator $\mathcal{U}($ resp. $\hat{\mathcal{U}})$ is a differential operator quadratic in the $t_{p}^{k}$ 's and $\frac{\partial}{\partial t_{p}^{k}}$ (resp. the $T_{p}^{k}$ 's and $\left.\frac{\partial}{\partial T_{p}^{k}}\right)$.

In order to get a glimpse of the difference between these two decompositions we give some more details (everything and much more can be found in the literature). A spectral curve $S$ is a compact Riemman surface $\Xi$ endowed with two meromorphic functions ${ }^{2} x, y \in$ $\mathcal{M}(\Xi)$ satisfying an algebraic equation $S(x, y)=0 .{ }^{3}$ One defines the 1 -form $\omega_{1}^{0}=y d x$ on

\footnotetext{
${ }^{1}$ I.e. invariant through symplectomorphism of the spectral curve. This definition actually corresponds to a cohomological field theory.

${ }^{2}$ In the case of meromorphic functions the spectral curve is said to be algebraic. Moreover if $\Xi$ is of genus 0 it is rational.

${ }^{3}$ Exists because of the algebraic character of the spectral curve.
} 
$\Xi$ and $\omega_{2}^{0}=B$ is the Bergmann kernel of $\Xi$ (we need a choice of polarization of $\Xi$ to define everything properly). Denote the poles of $\omega_{1}^{0}$ by $\left\{\alpha_{i}\right\}$ and its branch points by $\left\{a_{i}\right\}$. The operator $U$ can be computed by decomposing the global Virasoro constraints (equivalently the "loop equations") locally on the poles of $\omega_{1}^{0}$ leading to the results of [18], on the other hand this decomposition can be performed locally around the zeroes of $\omega_{1}^{0}$, this leads to the expression of $\hat{U}$ used to decompose on Kontsevitch tau functions. More formally the loop equations can be rewritten as:

$$
\mathcal{L}(p) \mathcal{Z}=0, \forall p \in \mathcal{C} .
$$

The construction of the $\mathcal{L}(p)$ being ensured by the data given above. In the limit $p \rightarrow \alpha_{i}$ these operators project onto local operators that can be described as:

$$
\mathcal{L}(p) \sim \sum_{n=1}^{\infty} \frac{d z_{i}(p)}{z_{i}(p)^{n+1}} L_{n}^{i}
$$

The $z_{i}$ 's being local coordinates around the poles $\alpha_{i}$. With the Virasoro operators $L_{n}^{i}$ taking the usual form for Hermitian 1-matrix models:

$$
L_{n}^{i}=\frac{1}{N^{2}}\left(2 n \frac{\partial}{\partial t_{n}^{i}}+\sum_{k=1}^{n-1} \frac{\partial}{\partial t_{n-k}^{i} \partial t_{k}^{i}}\right)+\sum_{p=1}^{d_{i}}(p+n) t_{n}^{i} \frac{\partial}{\partial t_{n+p}^{i}} .
$$

Analogously the projection can be made onto local operators defined around the branch points $\left\{a_{i}\right\}$. These operators are described in the formula (11) of [5].

\section{Tensor models}

In this section we introduce briefly the general framework of tensor models. These models have been introduced in the 90's in order to mimic the success of matrix models in more than two dimensions. In particular they have been constructed in order to give a definition of a 'sum over geometries' for three and more dimensions. Unfortunately they were at that time very difficult to handle analytically and the problem of generating well controlled triangulations was not understood [28]. Tensor models was then abandoned. Recently, Razvan Gurau revived interest in tensor models by constructing a (colored) model generating controlled triangulations. He was then able to construct a $1 / N$ expansion. The original point of view evolved after the 'uncoloring' paper [35]. For more details one can look in general references on the subject, for instance the necessary background is contained in $[27,35]$.

\subsection{Tensor invariants and generic 1-tensor model}

The uncolored point of view can be described as follows. The action of tensor model should be univalued when seen as a function on the vector space of tensor. Consequently it has to be constructed out of tensor invariants, in fact this is how matrix models are constructed, the trace being the invariant. 
First we shortly introduce the tensor invariants. To this aim we consider the tensors as multilinear forms on a direct product of vector spaces. A really nice and more detailed presentation of them is done in the second section of [20]. Consider a Hermitian space $(V, h)$ of complex dimension $\operatorname{dim} V=N, h$ being the Hermitian product on $V$. $h$ induces the usual isomorphism $V \rightarrow V *$ by $v \mapsto v *=h(v,$.$) . Denoting \left\{u_{i}\right\}_{i=1 \ldots N}$ a basis of $V$ and the dual basis $\left\{h\left(u_{i}, .\right)=\tilde{u}_{i}\right\}_{i=1 \ldots N}$, the coordinates of a vector in $V$ and its dual in $V *$ are related by complex conjugation from the property of the Hermitian product:

$$
v *=h(v, .)=h\left(\sum_{i} v_{i} u_{i}, .\right)=\sum_{i} \bar{v}_{i} \tilde{u}_{i} .
$$

A rank $D$ tensor $T$ is a multilinear form $T: V^{\times D} \rightarrow \mathbb{C}$, one can write in a basis:

$$
T=\sum_{\left\{i_{p}\right\}_{p=1 \ldots D}} T_{i_{1} \ldots i_{D}} \tilde{u}_{i_{1}} \otimes \ldots \otimes \tilde{u}_{i_{D}}
$$

Moreover the dual (denoted $\bar{T}$ ) of $T$ (i.e. the multilinear form on $V *^{\times D}$ ) is written in the basis $\left\{u_{i}\right\}_{i=1 \ldots N}$ :

$$
\bar{T}=\sum_{\left\{i_{p}\right\}_{p=1 \ldots D}} \bar{T}_{i_{1} \ldots i_{D}} u_{i_{1}} \otimes \ldots \otimes u_{i_{D}}
$$

by the property of the induced Hermitian product on a tensor product of Hermitian spaces.

By invariants of tensor we actually mean that the constructed quantity is invariant under any change of basis of $V$ and $V *$. If we change the basis by an element $g^{-1} \in$ $G L(N, \mathbb{C})$ the coordinates of a vector $v$ are changed by the matrix $\mathrm{U}(g)$ of $g$ and the coordinates of the dual vector are changed by $g^{-1}$. This induces the change of basis in the tensor product space, and thus on tensors:

$$
\begin{aligned}
T_{i_{1} \cdots i_{D}}^{\prime} & =\sum_{j_{1} \cdots j_{D}} \mathrm{U}\left(g_{1}^{-1}\right)_{i_{1} j_{1}} \mathrm{U}\left(g_{2}^{-1}\right)_{i_{2} j_{2}} \cdots \mathrm{U}\left(g_{D}^{-1}\right)_{i_{D} j_{D}} T_{j_{1} \cdots j_{D}} \\
\bar{T}_{i_{1} \ldots i_{D}}^{\prime} & =\sum_{j_{1} \cdots j_{D}} \mathrm{U}\left(g_{1}\right)_{i_{1} j_{1}} \mathrm{U}\left(g_{2}\right)_{i_{2} j_{2}} \cdots \mathrm{U}\left(g_{D}\right)_{i_{D} j_{D}} \bar{T}_{i_{1} \ldots i_{D}}
\end{aligned}
$$

This observation allows us to describe the possible tensor invariants. The invariants of order $2 p$ are $p$-linear in both $T$ and $\bar{T}$. Using the transformation rule given above, one notices that the only requirement for the quantity to be invariant is that the indices of a $T$ contract to the indices of a $\bar{T}$ with respect to their positions. The first index of a $T$ contracts with the first index of a $\bar{T}$ and so on. Thus to describe an invariant of order $2 p$ one only has to describe the contraction pattern of the $p T$ 's with the $p \bar{T}$ 's. This can be represented by bipartite graphs with colored edges. Indeed, if one represents the T's (resp. $\bar{T}$ 's) by white (resp. black) vertices with $D$ half-edges indexed with color labels from 1 to $D$. These labels represent the positions of the indices of the $T$ 's and $\bar{T}$ 's. Then the contraction of the $j^{t h}$ index of a $T$ with the $j^{t h}$ of a $\bar{T}$ is pictured by contracting the respecting half-edges in the graph. Thus the set of invariants of order $2 p$ are represented by all bipartite regular graphs of valence $D$ with a proper $D$-coloration of the edges whose examples are pictured on figure 1 . For any $D$-colored graph we define its jackets. 


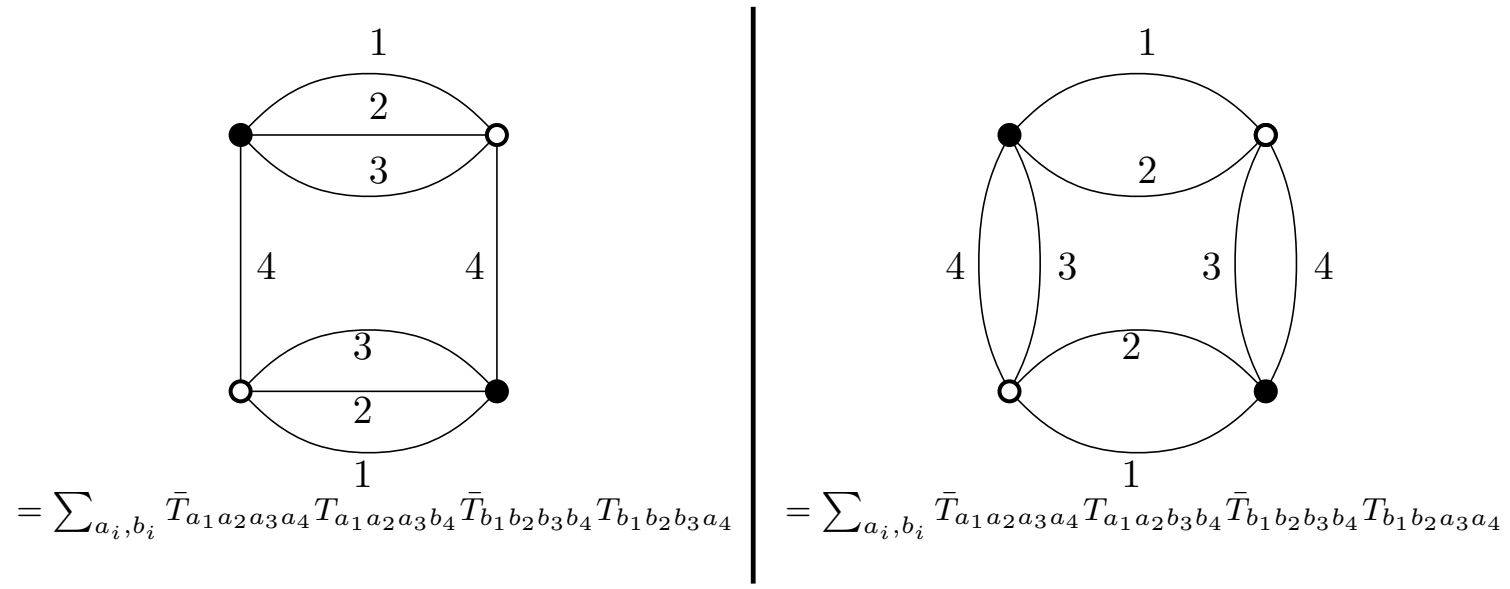

Figure 1. Two examples of tensor invariants for a rank 4 tensor.

Definition 3. A colored jacket $\mathcal{J}$ is a ribbon graph associated to a $D$-colored graph $\mathcal{G}$ with 1 -skeleton the graph $\mathcal{G}$ and faces made of graph cycles of colors $\left(\tau^{q}(0), \tau^{q+1}(0)\right)$ for $\tau \in \mathcal{S}_{D}$ a cyclic permutation, modulo the orientation of the cycle (i.e. $\tau^{-1}$ leads to the same jacket).

From this we define the degree:

Definition 4. The degree $\omega:\{D$-colored graphs $\} \rightarrow \mathbb{N}$ associates a positive integer to a $D$-colored graph $\mathcal{G}$ by:

$$
\omega(\mathcal{G})=\sum_{\mathcal{J}(\mathcal{G})} g_{\mathcal{J}}
$$

i.e. it is the sum of the genera of all the jackets $\mathcal{J}$ of $\mathcal{G}$.

We are now ready to define the generic tensor model.

Definition 5. The generic tensor model of dimension $D+1$ is defined by the partition function

$$
Z\left[N,\left\{t_{\mathcal{B}}\right\}\right]=\int d T d \bar{T} \exp \left(-N^{D-1} \sum_{\mathcal{B}} N^{-\frac{2}{(D-2) !} \omega(\mathcal{B})} t_{\mathcal{B}} \mathcal{B}(T, \bar{T})\right)
$$

where $\mathcal{B}$ runs over the regular $D$-colored graphs indexing the invariants. The $t_{\mathcal{B}}$ are the coupling constants, the one corresponding to the only invariant of order 2 often being fixed to one-half. $\mathcal{B}(\cdot, \cdot)$ being the invariant of $T$ and $\bar{T}$ indexed by the graph $\mathcal{B} . \omega(\mathcal{B})$ is the degree of $\mathcal{B}$.

One notices that the jackets are specifics. In fact in the 4-colored graphs case they provide cellular decompositions of Heegaard surfaces ${ }^{4}$ of the 3 -manifold represented by

\footnotetext{
${ }^{4}$ If one wants to be precise, one has to say that it gives a spine in general because when the triangulated object is a pseudo-manifold any neighborhood of a point does not have the topology of a ball.
} 
the graph [33]. Furthermore one can define a formal $1 / N$ expansion in the case of tensor models. Defining $F\left[N,\left\{t_{\mathcal{B}}\right\}\right]$ as $Z\left[N,\left\{t_{\mathcal{B}}\right\}\right]=\exp \left(-F\left[N,\left\{t_{\mathcal{B}}\right\}\right]\right)$ the expansion has this form

$$
F\left[N,\left\{t_{\mathcal{B}}\right\}\right]=\sum_{\omega \geq 0} N^{D-\frac{2}{(D-1) !} \omega} F_{\omega}\left[\left\{t_{\mathcal{B}}\right\}\right]
$$

where we are making some abuse of notations by writing $\omega$ as the sum index instead of the function defined on $(D+1)$-colored graphs taking integer values.

\section{2 $\quad T^{4}$ tensor models and intermediate field representation}

In this section we study the melonic $T^{4}$ (already studied in $[9,40]$ ) and write its intermediate field representation using hermitian matrices.

First we introduce some notations. Call $\mathcal{C}$ the set of colors, or equivalently the set that labels the positions of the indices of the components of the tensors. Since a $D$-dimensional tensor model is defined by the use of rank $D$ tensor, $|\mathcal{C}|=D$. For instance, for a tensor of rank three $\mathcal{C}=\{1,2,3\}$, each element of $\mathcal{C}$ indexes respectively the first, the second and the third index of the tensor. Moreover we introduce a partial Hermitian product notation. Consider a subset of $\mathcal{D} \subset \mathcal{C}$, we denote $\bar{T} \cdot \mathcal{D} T$ the contraction of all the indices labelled by elements in $\mathcal{D}, \bar{T} \cdot T$ denotes the contraction of all indices. And we denote by $\hat{i}$ the set $\mathcal{C}-\{i\}$.

In three dimensions using these notations one writes the quartic melonic interaction terms as:

$$
\begin{aligned}
V[\bar{T}, T] & =\sum_{a=1}^{D=3}(\bar{T} \cdot \hat{a} T) \cdot a(\bar{T} \cdot \hat{a} T) \\
& =\sum_{\text {all index }} \bar{T}_{s j k} T_{s^{\prime} j k} \bar{T}_{s^{\prime} j^{\prime} k^{\prime}} T_{s j^{\prime} k^{\prime}}+\bar{T}_{i s k} T_{i s^{\prime} k} \bar{T}_{i^{\prime} s^{\prime} k^{\prime}} T_{i^{\prime} s k^{\prime}}+\bar{T}_{i j s} T_{i j s^{\prime}} \bar{T}_{i^{\prime} j^{\prime} s^{\prime}} T_{i^{\prime} j^{\prime} s}
\end{aligned}
$$

By using this notation scheme we write the partition function of the quartic melonic tensor models in $D$-dimensions as:

$$
Z[\lambda, N]=\int d T d \bar{T} \exp \left[N^{D-1}\left(-\frac{1}{2}(\bar{T} \cdot T)-\frac{\lambda}{4} \sum_{a=1}^{D}\left(\bar{T} \cdot{ }_{a} T\right) \cdot{ }_{a}\left(\bar{T} \cdot{ }_{a} T\right)\right)\right] .
$$

It can be rewritten using intermediate field as:

$$
\begin{aligned}
Z[\lambda, N]= & \int \prod_{k} d \sigma_{k} d \bar{\sigma}_{k} \exp \left[-\frac{N}{2} \sum_{c=1 \ldots D} \operatorname{Tr}\left(\sigma_{c}^{2}\right)\right. \\
& \left.-\operatorname{Tr} \log \left(\mathbb{1}^{\otimes D}+i \sqrt{\frac{\lambda}{2 N^{D-2}}} \sum_{c=1 \ldots D} \mathbb{1}^{\otimes(c-1)} \otimes \sigma_{c} \otimes \mathbb{1}^{\otimes(D-c)}\right)\right] .
\end{aligned}
$$

One obtains this representation by writing the $T^{4}$ interaction term as:

$$
\begin{aligned}
& \exp \left[-N^{D-1} \frac{\lambda}{4}(\bar{T} \cdot \hat{a} T) \cdot{ }_{a}(\bar{T} \cdot \hat{a} T)\right] \\
& \quad=\int d \sigma_{a} d \bar{\sigma}_{a} \exp \left[-\frac{N}{2} \operatorname{Tr}\left(\sigma_{a}^{2}\right)-i \sqrt{N^{D} \lambda / 2} \operatorname{Tr}\left(\Theta^{a} \sigma_{a}\right)\right],
\end{aligned}
$$


where $\Theta^{a}$ denotes $\bar{T} \cdot \hat{a} T$. Then by replacing the interaction terms of (3.10) by the right hand side of eq. (3.12), we get:

$$
\begin{aligned}
Z[\lambda, N]= & \int d T d \bar{T} \prod_{a} d \sigma_{a} d \bar{\sigma}_{a} \exp \left[N^{D-1}\left(-\frac{1}{2}(\bar{T} \cdot T)\right)\right. \\
& \left.-\frac{N}{2} \sum_{a} \operatorname{Tr}\left(\sigma_{a}^{2}\right)-i \sqrt{N^{D} \lambda / 2} \operatorname{Tr}\left(\Theta^{a} \sigma_{a}\right)\right] .
\end{aligned}
$$

Integrating out the tensor $\bar{T}, T$ fields we end with:

$$
\begin{aligned}
Z[\lambda, N]= & \int \prod_{a} d \bar{\sigma}_{a} d \sigma_{a} \operatorname{det}\left[\mathbb{1}^{\otimes D}+i \sqrt{\frac{\lambda}{2 N^{D-2}}} \sum_{c=1 \ldots D} \mathbb{1}^{\otimes(c-1)} \otimes \sigma_{c} \otimes \mathbb{1}^{\otimes(D-c)}\right]^{-1} \\
& \exp \left[-\frac{N}{2} \sum_{a} \operatorname{Tr}\left(\sigma_{a}^{2}\right)\right]
\end{aligned}
$$

And so one obtains eq. (3.11) by writing the determinant in (3.14) as

$$
\begin{aligned}
\operatorname{det} & {\left[\mathbb{1}^{\otimes D}+i \sqrt{\frac{\lambda}{2 N^{D-2}}} \sum_{c=1 \ldots D} \mathbb{1}^{\otimes(c-1)} \otimes \sigma_{c} \otimes \mathbb{1}^{\otimes(D-c)}\right] } \\
= & \exp \left[\operatorname{Tr} \log \left(\mathbb{1}^{\otimes D}+i \sqrt{\frac{\lambda}{2 N^{D-2}}} \sum_{c=1 \ldots D} \mathbb{1}^{\otimes(c-1)} \otimes \sigma_{c} \otimes \mathbb{1}^{\otimes(D-c)}\right)\right] .
\end{aligned}
$$

One notices that expanding the logarithm of (3.11) the matrix model one obtains looks like the matrix models introduced in [6] for specific choices of the values of the formal variables. The models introduced therein are studied because of their connection to LMO invariants of 3-manifolds (see [7]). The study of possible connections to tensor models could be interesting and the subject of further works.

\section{Constructing $T^{4}$ tensor model out of matrix models}

In this section we construct the $T^{4}$ tensor models as an action of a differential operator on a product of Hermitian 1-matrix model. Starting from the intermediate field representation we show:

Theorem 1. The partition function of the D-dimensional melonic $T^{4}$ model can be rewritten as:

$$
Z[\lambda, N]=e^{\hat{X}} e^{\hat{Y}} \prod_{i=1}^{D} Z_{1 M M}\left[\left\{t_{p}^{i}\right\}_{p \in \mathbb{N}}\right]=e^{\hat{\mathcal{O}}} \prod_{i=1}^{D} Z_{1 M M}^{i}\left[\left\{t_{p}^{i}\right\}_{p \in \mathbb{N}}\right]
$$

where $Z_{1 M M}\left[\left\{t_{p}^{i}\right\}_{p \in \mathbb{N}}\right]$ is a Hermitian 1-matrix model partition function and $\hat{X}, \hat{Y}, \hat{\mathcal{O}}$ are differential operators acting on the times $t_{p}^{i}$ (or coupling constants) of the 1-matrix model:

$$
\begin{aligned}
& \hat{X}=-\sum_{i, p} t_{p}^{i} \frac{\partial}{\partial t_{p}^{i}} \\
& \hat{Y}=\frac{(-1)^{D}}{N^{D}} \sum_{\left(q_{1}, \ldots, q_{D}\right) \in\left(\mathbb{N}^{D}\right)^{*}} \frac{(-i)^{\sum q_{i}}}{\sum q_{i}}{\sqrt{\frac{\lambda}{2 N^{D-2}}}}^{q_{1}+\ldots+q_{D}}\left(\begin{array}{c}
q_{1}+\ldots+q_{D} \\
q_{1}, \ldots, q_{D}
\end{array}\right) \frac{\partial^{D}}{\partial t_{q_{1}}^{1} \ldots \partial t_{q_{D}}^{D}}
\end{aligned}
$$


and

$$
\hat{\mathcal{O}}=\ln \left(e^{\hat{X}} e^{\hat{Y}}\right)=\hat{X}+\frac{D}{2} \frac{\exp (D / 2)}{\sinh (D / 2)} \hat{Y}
$$

Proof. In order to prove this, we make use of the intermediate field representation of the $T^{4}$ tensor model.

$$
\begin{aligned}
Z_{T_{m}^{4}}[\lambda, N] & =\int \prod_{k} d \sigma_{k} d \overline{\sigma_{k}} \\
& \exp \left[-\frac{N}{2} \sum_{c=1 \ldots D} \operatorname{Tr}\left(\sigma_{c}^{2}\right)-\operatorname{Tr} \ln \left(\mathbb{1}^{\otimes D}+i \sqrt{\frac{\lambda}{N^{D-2}}} \sum_{c=1 \ldots D} \mathbb{1}^{\otimes(c-1)} \otimes \sigma_{c} \otimes \mathbb{1}^{\otimes(D-c)}\right)\right] .
\end{aligned}
$$

Taylor expanding the logarithmic potential in $\sqrt{\lambda}$ we get:

$$
\begin{aligned}
Z_{T_{m}^{4}}[\lambda, N]= & \int \prod_{k} d \sigma_{k} d \overline{\sigma_{k}} \exp \left[-\frac{N}{2} \sum_{c=1 \cdots D} \operatorname{Tr}\left(\sigma_{c}^{2}\right)\right] \\
& \exp \left[\sum_{p>0}-\operatorname{Tr}\left(\frac{(-i)^{p}}{p}{\sqrt{\frac{\lambda}{2 N^{D-2}}}}^{p}\left(\sum_{c=1 \ldots D} \mathbb{1}^{\otimes(c-1)} \otimes \sigma_{c} \otimes \mathbb{1}^{\otimes(D-c)}\right)^{p}\right)\right] .
\end{aligned}
$$

Using multinomial coefficients we can expand $\operatorname{Tr}\left(\sum_{c=1 \cdots D} \mathbb{1}^{\otimes(c-1)} \otimes \sigma_{c} \otimes \mathbb{1}^{\otimes(D-c)}\right)^{p}$, and so we obtain:

$$
\begin{aligned}
Z_{T_{m}^{4}}[\lambda, N]= & \int \prod_{k} d \sigma_{k} d \bar{\sigma}_{k} \exp \left[-\frac{1}{2} \sum_{c=1 \ldots D} \operatorname{Tr}\left(\sigma_{c}^{2}\right)\right] \\
& \exp \left[\sum_{\left(q_{1}, \ldots, q_{D}\right) \in\left(\mathbb{N}^{D}\right)^{*}} \frac{(-i)^{\sum q_{i}}}{\sum q_{i}}{\sqrt{\frac{\lambda}{2 N^{D-2}}}}^{\sum q_{i}}\left(\begin{array}{c}
\sum_{i} q_{i} \\
q_{1}, \ldots, q_{D}
\end{array}\right) \prod_{c=1}^{D} \operatorname{Tr}\left(\sigma_{c}^{q_{c}}\right)\right] .
\end{aligned}
$$

Noticing that for a generic Hermitian 1-matrix model we have the identity:

$$
\frac{\partial}{\partial t_{p}} Z_{1 M M}\left[\left\{t_{p}\right\}\right]=-N\left\langle\operatorname{Tr}\left(\sigma^{p}\right)\right\rangle_{\mathrm{int}}
$$

We can represent the $T^{4}$ partition function since in fact:

$$
\begin{aligned}
Z_{T_{m}^{4}}[\lambda, N] & =\prod_{k} d \sigma_{k} d \overline{\sigma_{k}} \exp \left[-\frac{1}{2} \sum_{c=1 \ldots D} \operatorname{Tr}\left(\sigma_{c}^{2}\right)\right] \\
& \sum_{n \geq 0} \frac{1}{n !}\left[\sum_{\left(q_{1}, \ldots, q_{D}\right) \in\left(\mathbb{N}^{D}\right)^{*}} \frac{(-i)^{\sum q_{i}}}{\sum q_{i}}{\sqrt{\frac{\lambda}{2 N^{D-2}}}}^{\sum q_{i}}\left(\begin{array}{c}
\sum_{i} q_{i} \\
q_{1}, \ldots, q_{D}
\end{array}\right) \prod_{c=1}^{D} \operatorname{Tr}\left(\sigma_{c}^{q_{c}}\right)\right]^{n} \\
= & \left\langle\sum_{n \geq 0} \frac{1}{n !}\left[\sum_{\left(q_{1}, \ldots, q_{D}\right) \in\left(\mathbb{N}^{D}\right)^{*}} \frac{(-i)^{\sum q_{i}}}{\sum q_{i}}{\sqrt{\frac{\lambda}{2 N^{D-2}}}}^{q_{i}}\left(\begin{array}{c}
\sum_{i} q_{i} \\
q_{1}, \cdots, q_{D}
\end{array}\right) \prod_{c=1}^{D} \operatorname{Tr}\left(\sigma_{c}^{q_{c}}\right)\right]^{n}\right\rangle_{\operatorname{gau} \beta}
\end{aligned}
$$


Using equation (4.8) one can write similar correlation functions by differentiating product of matrix models:

$$
\begin{aligned}
& \left\langle\frac{(-i)^{\sum q_{i}}}{\sum q_{i}}{\sqrt{\frac{\lambda}{2 N^{D-2}}}}^{\sum q_{i}}\left(\begin{array}{c}
\sum_{i} q_{i} \\
q_{1}, \ldots, q_{D}
\end{array}\right) \prod_{c=1}^{D} \operatorname{Tr}\left(\sigma_{c}^{q_{c}}\right)\right\rangle_{\mathrm{int}} \\
& \quad=\frac{(-1)^{D}(-i)^{\sum q_{i}}}{N^{D} \sum q_{i}}{\sqrt{\frac{\lambda}{2 N^{D-2}}}}^{\sum q_{i}}\left(\begin{array}{c}
\sum_{i} q_{i} \\
q_{1}, \ldots, q_{D}
\end{array}\right) \frac{\partial^{D}}{\partial t_{q_{1}}^{1} \partial t_{q_{2}}^{2} \ldots \partial t_{q_{D}}^{D}} \prod_{i=1}^{D} Z_{1 M M}\left[\left\{t_{p}^{i}\right\}_{p=0}^{\infty}\right] .
\end{aligned}
$$

It follows:

$$
\begin{aligned}
& \left\langle\sum_{n \geq 0} \frac{1}{n !}\left[\sum_{\left(q_{1}, \ldots, q_{D}\right) \in\left(\mathbb{N}^{D}\right)^{*}} \frac{(-i)^{\sum q_{i}}}{\sum q_{i}}{\sqrt{\frac{\lambda}{2 N^{D-2}}}}^{\sum q_{i}}\left(\begin{array}{c}
\sum_{i} q_{i} \\
q_{1}, \cdots, q_{D}
\end{array}\right) \prod_{c=1}^{D} \operatorname{Tr}\left(\sigma_{c}^{q_{c}}\right)\right]^{n}\right\rangle_{\mathrm{int}} \\
& =\exp \left[\sum_{\left(q_{1}, \ldots, q_{D}\right) \in\left(\mathbb{N}^{D}\right)^{*}} \frac{(-1)^{D}(-i)^{\sum q_{i}}}{N^{D} \sum q_{i}}{\sqrt{\frac{\lambda}{2 N^{D-2}}}}^{\sum q_{i}}\left(\begin{array}{c}
\sum_{i} q_{i} \\
q_{1}, \ldots, q_{D}
\end{array}\right) \frac{\partial^{D}}{\partial t_{q_{1}}^{1} \partial t_{q_{2}}^{2} \ldots \partial t_{q_{D}}^{D}}\right] \prod_{i=1}^{D} Z^{i}\left[\left\{t_{p}^{i}\right\}_{p=0}^{\infty}\right],
\end{aligned}
$$

since the differential operators commute. One defines $\hat{Y}$ by:

$$
\hat{Y}=\sum_{\left(q_{1}, \ldots, q_{D}\right) \in\left(\mathbb{N}^{D}\right)^{*}} \frac{(-1)^{D}(-i)^{\sum q_{i}}}{N^{D} \sum q_{i}}{\sqrt{\frac{\lambda}{2 N^{D-2}}}}^{\sum q_{i}}\left(\begin{array}{c}
\sum_{i} q_{i} \\
q_{1}, \ldots, q_{D}
\end{array}\right) \frac{\partial^{D}}{\partial t_{q_{1}}^{1} \partial t_{q_{2}}^{2} \ldots \partial t_{q_{D}}^{D}} .
$$

In order to obtain the Gaussian expectation values, and not the interacting one, we act with another operator whose role is to suppress the original matrix potential. In this manner we get the $T^{4}$ partition function. Define $\hat{X}$ by:

$$
\hat{X}=-\sum_{i=1}^{D} \sum_{p=0}^{\infty} t_{p}^{i} \frac{\partial}{\partial t_{p}^{i}}
$$

acting with $\exp (\hat{X})$ on $\exp (\hat{Y}) \prod_{i} Z_{1 M M}\left[\left\{t_{p}^{i}\right\}_{p=0}^{\infty}\right]$ suppresses the matrix potential term as shown by a direct computation. One wants to find an explicit form for $\hat{\mathcal{O}}$, the commutator of $\hat{X}$ and $\hat{Y}$ is given by:

$$
\begin{aligned}
{[\hat{X}, \hat{Y}] } & =\sum_{\left(q_{1}, \ldots, q_{D}\right) \in(\mathbb{N} D)^{*}} \frac{(-1)^{D}(-i)^{\sum q_{i}}}{N^{D} \sum q_{i}} \sqrt{\frac{\lambda}{2 N^{D-2}}}{ }^{\sum q_{i}}\left(\begin{array}{c}
\sum_{i} q_{i} \\
q_{1}, \ldots, q_{D}
\end{array}\right) \frac{\partial^{D}}{\partial t_{q_{1}}^{1} \partial t_{q_{2}}^{2} \ldots \partial t_{q_{D}}^{D}} \sum_{i=1}^{D} \sum_{p=0}^{\infty} t_{p}^{i} \frac{\partial}{\partial t_{p}^{i}} \\
& =\sum_{\left(q_{1}, \ldots, q_{D}\right) \in\left(\mathbb{N}^{D}\right)^{*}} \sum_{i=1}^{D} \sum_{p=0}^{\infty} \frac{(-1)^{D}(-i)^{\sum q_{i}}}{N^{D} \sum q_{i}}{\sqrt{\frac{\lambda}{2 N^{D-2}}}}^{\sum q_{i}}\left(\begin{array}{c}
\sum_{i} q_{i} \\
q_{1}, \ldots, q_{D}
\end{array}\right) \sum_{j} \delta^{j i} \delta_{p q_{j}} \frac{\partial^{D}}{\partial t_{q_{1}}^{1} \partial t_{q_{2}}^{2} \ldots \partial t_{q_{D}}^{D}} \\
& =\sum_{\left(q_{1}, \ldots, q_{D}\right) \in\left(\mathbb{N}^{D}\right)^{*}} \sum_{i=1}^{D} \frac{(-1)^{D}(-i)^{\sum q_{i}}}{N^{D} \sum q_{i}}{\sqrt{\frac{\lambda}{2 N^{D-2}}}}^{\sum q_{i}}\left(\begin{array}{c}
\sum_{i} q_{i} \\
q_{1}, \ldots, q_{D}
\end{array}\right) \frac{\partial^{D}}{\partial t_{q_{1}}^{1} \partial t_{q_{2}}^{2} \ldots \partial t_{q_{D}}^{D}} \\
& =D \sum_{\left(q_{1}, \ldots, q_{D}\right) \in\left(\mathbb{N}^{D}\right)^{*}} \frac{(-1)^{D}(-i)^{\sum q_{i}}}{N^{D} \sum q_{i}}{\sqrt{\frac{\lambda}{2 N^{D-2}}}}^{\sum q_{i}}\left(\begin{array}{c}
\sum_{i} q_{i} \\
q_{1}, \ldots, q_{D}
\end{array}\right) \frac{\partial^{D}}{\partial t_{q_{1}}^{1} \partial t_{q_{2}}^{2} \ldots \partial t_{q_{D}}^{D}}=D \hat{Y} .
\end{aligned}
$$




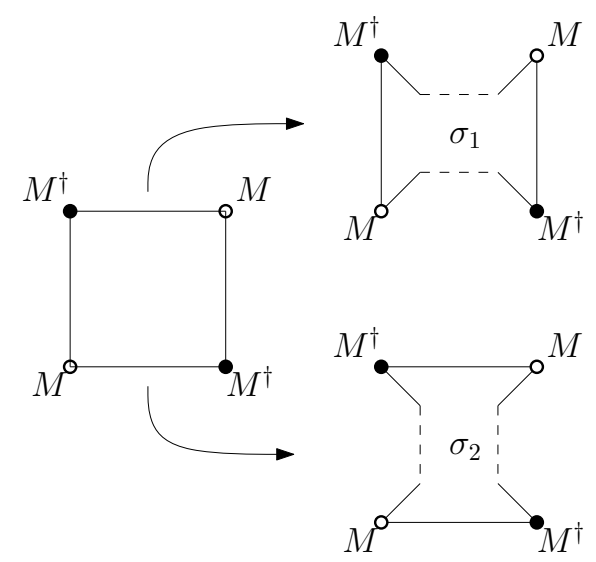

Figure 2. This figure shows the splitting when we consider the quartic melonic tensor model of rank 2. It collapses to a complex matrix model with complex matrix $M$. It can be splitted with two Hermitian matrices $\sigma_{1}, \sigma_{2}$.

Therefore using Hausdorff-Baker-Campbell formula we can find an explicit form for the operator $\hat{\mathcal{O}}$ :

$$
\hat{\mathcal{O}}=\log \left[e^{\hat{X}} e^{\hat{Y}}\right]=\hat{X}+\frac{D}{1-\exp (-D)} \hat{Y}=\hat{X}+\frac{D}{2} \frac{\exp (D / 2)}{\sinh (D / 2)} \hat{Y}
$$

Paying more attention to the operators $\hat{X}$ and $\hat{Y}$ one notices that they span an Aff(1) Lie algebra. We notice that this differential operator is not quadratic in the $\frac{\partial}{\partial t_{q_{i}}^{i}}$ and so this is not a Givental decomposition. However we can reproduce this decomposition in two cases,

- in the case of rank 2 tensor models, the quartic melonic model collapses onto a complex matrix model with a $\operatorname{Tr}\left(M M^{\dagger} M M^{\dagger}\right)$. By splitting this interaction in two ways with two Hermitian matrices $\sigma_{1}, \sigma_{2}$ (see figure 2), we get a model with a $\operatorname{Tr} \log \left(\mathbb{1}^{\otimes 2}+i \sqrt{\lambda / 2}\left(\sigma_{1} \otimes \mathbb{1}+\mathbb{1} \otimes \sigma_{2}\right)\right)$ interaction. $^{5}$ This can be represented by a quadratic $\hat{Y}$ operator so that this case reduces to a Givental decomposition.

- Also one can reduce the number of interaction terms for the quartic tensor models of any ranks. If this number of interactions reduces to two, we also get a Givental decomposition.

\section{$5 \quad$ Bilinear identities for $T^{4}$ tensor model}

We begin by introducing the orthogonal polynomials for the 1-Hermitian matrix model, we follow closely the presentation given by [13]. For each value of the coupling constant $\vec{t}=\left(t_{p}\right)_{p=0 \cdots \infty}$ and of $N$ the size of the matrix we define:

\footnotetext{
${ }^{5} \mathrm{Up}$ to symmetry factors.
} 
Definition 6. The family of orthogonal polynomials parametrized by $N$ and $\left\{t_{i}\right\}$ in the variable $x$ for the matrix measure is defined by:

$$
P_{N, \vec{t}}=\langle\operatorname{det}(x-M)\rangle_{N, \vec{t}}
$$

i.e. the mean value of the characteristic polynomial of the matrix.

These polynomials are orthogonal to the matrix measure defined by the partition function of the 1-Hermitian matrix model. This is only dependant on the fact that the measure comes with a Vandermonde determinant when written in eigenvalues coordinates and that the interaction is symmetric in the coordinates.

For the Hermitian matrix model, the Hirota's equations amount to write the orthogonality relations for the characteristic polynomial. They can be written using vertex operators:

$$
\frac{1}{2 i \pi} \oint d z\left(V_{+}(z) Z_{1 M M}\left[\left\{t_{i}\right\}\right]\right)\left(V_{-}(z) Z_{1 M M}\left[\left\{\tilde{t}_{i}\right\}\right]\right)=0
$$

where $V_{ \pm}(z)=\exp \left( \pm \sum_{n \geq 0} z^{n} t_{n}\right) \exp \left(\mp \log \left(\frac{1}{z}\right) \frac{\partial}{N \partial t_{0}} \mp \sum_{n \geq 1} \frac{z^{-n}}{n} \frac{\partial}{N \partial t_{n}}\right)$. One would hope the integrable structure of the Hermitian matrix models used for the decomposition survives the action of $\exp (\hat{Y})$. In fact the bilinear Hirota's equations of the Hermitian 1-matrix model lead to a set of bilinear identities for the tensor model. Generalizing an idea of [13], one acts by conjugation on the vertex operators of each matrix model of color $c$ :

$$
V_{ \pm}^{c}(z, \lambda)=\exp (\hat{Y}) V_{ \pm}^{c}(z) \exp (-\hat{Y})
$$

in some sense we make the vertex operators evolve to the intermediate field representation of tensor model. ${ }^{6}$ Thus we obtain a set of identities of the form:

$$
\oint\left(V_{+}^{c}(z, \lambda) \exp (\hat{Y}) \prod_{c^{\prime}=1}^{D} Z_{1 M M}\left[\left\{t_{i}^{c^{\prime}}\right\}\right]\right)\left(V_{-}^{c}(z, \lambda) \exp (\hat{Y}) \prod_{c^{\prime}=1}^{D} Z_{1 M M}\left[\left\{\tilde{t}_{i}^{c^{\prime}}\right\}\right]\right)=0
$$

for each $c \in \llbracket 1, D \rrbracket$. Then one has to write these equations in term of the matrices. To this aim we compute the explicit $V_{ \pm}^{c}(z, \lambda)$. First set $\hat{A}^{c}=\sum_{p \geq 0} z^{p} t_{p}^{c}$ and $\hat{B}^{c}=\log \left(\frac{1}{z}\right) \frac{\partial}{N \partial t_{0}^{c}}+$ $\sum_{n=1}^{\infty} \frac{z^{-n}}{n} \frac{\partial}{N \partial t_{n}^{c}}$. Therefore, for $c \in \llbracket 1, D \rrbracket$ :

$$
\begin{aligned}
{\left[\hat{B}^{c}, \hat{Y}\right]=} & 0 \\
{\left[\hat{A}^{c}, \hat{Y}\right]=} & -\frac{(-1)^{D}}{N^{D}} \sum_{\left(q_{1}, \ldots, q_{D}\right) \in\left(\mathbb{N}^{D}\right)^{*}} \frac{(-i)^{\sum q_{i}}}{\sum q_{i}}{\sqrt{\frac{\lambda}{2 N^{D-2}}}}^{q_{1}+\ldots+q_{D}} \\
& \times\left(\begin{array}{c}
q_{1}+\ldots+q_{D} \\
q_{1}, \ldots, q_{D}
\end{array}\right) z^{q_{c}} \frac{\partial^{D-1}}{\partial t_{q_{1}}^{1} \ldots \partial \hat{t}_{q_{c}}^{c} \ldots \partial t_{q_{D}}^{D}}
\end{aligned}
$$

\footnotetext{
${ }^{6}$ It's not exactly the tensor model that one obtains, unless one sets the $t_{i}^{c}$, s to zero. But one can argue that these bilinear identities are satisfied whatever the value of the $t_{i}^{c}$ 's and thus induce bilinear identities for the corresponding tensor model.
} 
One computes the evolved operators explicitly:

$$
\begin{aligned}
V_{ \pm}^{c}(z, \lambda) & =\exp \left( \pm \hat{A}^{c}\right) \exp \left(\mp \hat{A}^{c}\right) \exp (\hat{Y}) \exp \left( \pm \hat{A}^{c}\right) \exp \left(\mp \hat{B}^{c}\right) \exp (-\hat{Y}) \\
& =\exp \left( \pm \hat{A}^{c}\right) \exp \left(e^{\mp a d_{\hat{A}}} \hat{Y}\right) \exp \left(\mp \hat{B}^{c}\right) \exp (-\hat{Y}) .
\end{aligned}
$$

Finally noting that $a d_{\hat{A}^{c}}^{n}(Y)=0$ for $n \geq 2$.

$$
\begin{aligned}
V_{ \pm}^{c}(z, \lambda) & =\exp \left( \pm \hat{A}^{c}\right) \exp \left(\hat{Y} \mp\left[\hat{A}^{c}, \hat{Y}\right]\right) \exp \left(\mp \hat{B}^{c}\right) \exp (-\hat{Y}) \\
& =\exp \left( \pm \hat{A}^{c}\right) \exp \left(\mp\left[\hat{A}^{c}, \hat{Y}\right]\right) \exp \left(\mp \hat{B}^{c}\right) .
\end{aligned}
$$

So one has proved the following proposition:

Proposition 1. The explicit form of the operators $V_{ \pm}^{c}(z, \lambda)$ for $c \in \llbracket 1, D \rrbracket$ is given by:

$$
\begin{aligned}
V_{ \pm}^{c}(z, \lambda)= & e^{ \pm \sum_{p=0}^{\infty} t_{p}^{c} z^{p}} e^{\mp \log \left(\frac{1}{z}\right) \frac{\partial}{N \partial t_{0}^{c}} \mp \sum_{n=0}^{\infty} \frac{z^{-n}}{n} \frac{\partial}{N \partial t_{n}^{c}}} \\
& e^{ \pm \frac{(-1) D}{N D} \sum_{\left(q_{1}, \ldots, q_{D}\right) \in(\mathbb{N} D)^{*}} \frac{(-i) \sum q_{i}}{\sum q_{i}}{\sqrt{\frac{\lambda}{2 N^{D-2}}}}^{q_{1}+\ldots+q_{D}}\left(\begin{array}{c}
q_{1}+\ldots+q_{1} \\
q_{1}, \ldots, q_{D}
\end{array}\right) z^{q_{c}} \frac{\partial^{D-1}}{\partial t_{q_{1}}^{1} \ldots \partial t_{q_{C}}^{c} \ldots \partial t_{q_{D}}^{D}}} .
\end{aligned}
$$

Using this proposition we get the form of the Hirota's equation for the intermediate field representation of the tensor model. These can be explicitly rewritten as:

$$
\begin{aligned}
0= & \oint d z e^{\sum_{n} z^{n}\left(t_{n}-\tilde{t}_{n}\right)} \\
& \left\langle\frac{\operatorname{det}\left(z-\sigma_{c}\right)}{\operatorname{det}\left(\mathbb{1}^{\otimes D}\left(1+z \sqrt{\frac{\lambda}{2 N^{D-2}}}\right)+\sqrt{\frac{\lambda}{2 N^{D-2}}} \sum_{i \neq c} \mathbb{1}^{\otimes(D-c)} \otimes \sigma_{c} \otimes \mathbb{1}^{\otimes(c-1)}\right)}\right\rangle_{N, t} \\
& \left\langle\frac{\operatorname{det}\left(\mathbb{1}^{\otimes D}\left(1+z \sqrt{\frac{\lambda}{2 N^{D-2}}}\right)+\sqrt{\frac{\lambda}{2 N^{D-2}}} \sum_{i \neq c} \mathbb{1}^{\otimes(D-c)} \otimes \sigma_{c} \otimes \mathbb{1}^{\otimes(c-1)}\right)}{\operatorname{det}\left(z-\sigma_{c}\right)}\right\rangle_{N^{\prime}, \tilde{t}} .
\end{aligned}
$$

We should be able to write these equations in terms of tensor variables. In fact in [45] we get a relation between powers of intermediate matrices and power of $\Theta_{c}$ matrices of the form:

$$
\sigma_{c}^{q}=H_{q}\left(\Theta^{c}\right),
$$

$H_{q}$ being the $q^{\text {th }}$ Hermite polynomial. We postpone this to future work.

\section{Conclusion}

In this paper we unravelled a decomposition of a specific tensor model by the mean of an intertwining operator acting on a product of Hermitian matrix models. The intertwining operator is not of a Givental type since it is not quadratic in the coupling constants. However it collapses to a Givental operator in specific cases. Rank 2 tensor models and quartic melonic tensor models with only two interactions are some of these cases. Furthermore we do know that the resulting partition functions count specific types of polyangulations of pseudo-manifolds in $D$ dimensions. Moreover this decomposition formula allowed to derive bilinear equations for the tensor models as a 'deformation' of Hirota's equations of the matrix models. 
It would be interesting to understand more about these specific tensor models especially in a more geometric fashion. Can this decomposition help to grasp the geometrical meaning of the number generated by the partition function of the model? Do the observables of this tensor models have anything to do with the symplectic invariants computed by the topological recursion? For some tensor models we know they can be written as matrix models in several way. Do tensor models provide a framework for writing matrix models satisfying duality relations between them? A related question being: is there any hope to write Givental models as some sort of tensor models? Also, is our model integrable and can we find any method to compute it exactly? Answering one or more of these questions could shed light on the real mathematical nature of these tensor models.

Moreover it should be investigated whether or not it is possible to generalize this decomposition to arbitrary tensor models in a natural way.

\section{Acknowledgments}

I am very grateful to Bertrand Eynard for numerous discussions about Givental decompositions, Topological Recursion and integrability in matrix models. I would also acknowledge Valentin Bonzom for fruitful exchanges about tensor models and integrability therein. I also thank Vincent Rivasseau for continously supporting me during this work. I thank an anonymous referee for its careful reading and pushing me to improve this manuscript. The Institut Galilée and LPT provide excellent working environment. Part of this work has been written at the Erwin Schrödinger Institute during the "Combinatorics, Geometry and Physics 2014" program in Vienna. S. Dartois is partially supported by the ANR JCJC CombPhysMat2Tens grant.

Open Access. This article is distributed under the terms of the Creative Commons Attribution License (CC-BY 4.0), which permits any use, distribution and reproduction in any medium, provided the original author(s) and source are credited.

\section{References}

[1] J. Ambjørn, B. Durhuus and T. Jonsson, Three-dimensional simplicial quantum gravity and generalized matrix models, Mod. Phys. Lett. A 6 (1991) 1133 [InSPIRE].

[2] N. Sasakura, Tensor model for gravity and orientability of manifold, Mod. Phys. Lett. A 6 (1991) 2613 [INSPIRE].

[3] M.L. Mehta, Random Matrices, Pure and Applied Mathematics 142 (2004), Elsevier/Academic Press, Amsterdam, Netherlands.

[4] P. Di Francesco, P.H. Ginsparg and J. Zinn-Justin, 2D Gravity and random matrices, Phys. Rept. 254 (1995) 1 [hep-th/9306153] [INSPIRE].

[5] A.S. Alexandrov, A. Mironov and A. Morozov, M-theory of matrix models, Theor. Math. Phys. 150 (2007) 153 [hep-th/0605171] [INSPIRE].

[6] G. Borot, Formal multidimensional integrals, stuffed maps and topological recursion, arXiv: 1307.4957 [INSPIRE]. 
[7] S. Garoufalidis and M. Mariño, On Chern-Simons matrix models, math/0601390 [INSPIRE].

[8] P. Di Francesco, 2D Quantum Gravity, Matrix Models and Graph Combinatorics, Nato Sci. Ser. II 221 (2006) 33 [math-ph/0406013] [INSPIRE].

[9] S. Dartois, R. Gurau and V. Rivasseau, Double Scaling in Tensor Models with a Quartic Interaction, JHEP 09 (2013) 088 [arXiv: 1307.5281] [INSPIRE].

[10] R. Gurau and G. Schaeffer, Regular colored graphs of positive degree, arXiv:1307.5279.

[11] V. Bonzom, R. Gurau, J.P. Ryan and A. Tanasa, The double scaling limit of random tensor models, JHEP 09 (2014) 051 [arXiv: 1404.7517] [INSPIRE].

[12] C. Itzykson and J.B. Zuber, The Planar Approximation. 2, J. Math. Phys. 21 (1980) 411 [INSPIRE].

[13] J. Alfaro and I.K. Kostov, Generalized Hirota equations in models of $2 D$ quantum gravity, hep-th/9604011 [INSPIRE].

[14] A. Givental, Semisimple Frobenius structures at higher genus, Int. Math. Res. Not. 2001 (2001) 1265 [math/0008067] [INSPIRE].

[15] A.B. Givental, Gromov-Witten invariants and quantization of quadratic hamiltonians, Moscow Math. J. 1 (2001) 551 [math/0108100] [INSPIRE].

[16] B. Eynard, Topological expansion for the 1-Hermitian matrix model correlation functions, JHEP 11 (2004) 031 [hep-th/0407261] [INSPIRE].

[17] B. Eynard and N. Orantin, Algebraic methods in random matrices and enumerative geometry, arXiv:0811.3531 [INSPIRE].

[18] N. Orantin, Symplectic invariants, Virasoro constraints and Givental decomposition, arXiv:0808.0635 [INSPIRE].

[19] P. Dunin-Barkowski, N. Orantin, S. Shadrin and L. Spitz, Identification of the Givental formula with the spectral curve topological recursion procedure, Commun. Math. Phys. 328 (2014) 669 [arXiv: 1211.4021] [INSPIRE].

[20] T. Delepouve, R. Gurau and V. Rivasseau, Universality and Borel Summability of Arbitrary Quartic Tensor Models, arXiv:1403.0170 [INSPIRE].

[21] D. Oriti, Group field theory as the 2nd quantization of Loop Quantum Gravity, arXiv:1310.7786 [INSPIRE].

[22] C. Rovelli, Quantum Gravity, Cambridge University Press, Cambridge, U.K. (2006).

[23] G. 't Hooft, A Planar Diagram Theory for Strong Interactions, Nucl. Phys. B 72 (1974) 461 [INSPIRE].

[24] W.T. Tutte, A census of planar triangulations, Canad. J. Math. 14 (1962) 21.

[25] V. Rivasseau, The Tensor Track, III, Fortsch. Phys. 62 (2014) 81 [arXiv:1311.1461] [INSPIRE].

[26] R. Gurau, Colored Group Field Theory, Commun. Math. Phys. 304 (2011) 69 [arXiv: 0907.2582] [INSPIRE].

[27] R. Gurau and J.P. Ryan, Colored Tensor Models - a review, SIGMA 8 (2012) 020 [arXiv: 1109.4812] [INSPIRE]. 
[28] R. Gurau, Lost in Translation: Topological Singularities in Group Field Theory, Class. Quant. Grav. 27 (2010) 235023 [arXiv: 1006.0714] [InSPIRE].

[29] M. Pezzana, Sulla struttura topologica delle varietà compatte, Atti Sem. Mat. Fis. Univ. Modena 23 (1974) 269.

[30] M. Ferri and C. Gagliardi, Crystallisation moves, Pacific J. Math. 100 (1982) 85.

[31] S. Lins, Gems, Computers, and Attractors for 3-manifolds, World Scientific, (1995).

[32] R. Gurau, The complete 1/N expansion of colored tensor models in arbitrary dimension, Annales Henri Poincaré 13 (2012) 399 [arXiv:1102.5759] [InSPIRE].

[33] J.P. Ryan, Tensor models and embedded Riemann surfaces, Phys. Rev. D 85 (2012) 024010 [arXiv:1104.5471] [INSPIRE].

[34] V. Bonzom, R. Gurau, A. Riello and V. Rivasseau, Critical behavior of colored tensor models in the large-N limit, Nucl. Phys. B 853 (2011) 174 [arXiv:1105.3122] [INSPIRE].

[35] V. Bonzom, R. Gurau and V. Rivasseau, Random tensor models in the large- $N$ limit: Uncoloring the colored tensor models, Phys. Rev. D 85 (2012) 084037 [arXiv:1202.3637] [INSPIRE].

[36] J. Ben Geloun and V. Rivasseau, A Renormalizable 4-Dimensional Tensor Field Theory, Commun. Math. Phys. 318 (2013) 69 [arXiv:1111.4997] [InSPIRE].

[37] S. Carrozza, Discrete Renormalization Group for SU(2) Tensorial Group Field Theory, arXiv: 1407.4615 [INSPIRE].

[38] V. Bonzom, R. Gurau and V. Rivasseau, The Ising Model on Random Lattices in Arbitrary Dimensions, Phys. Lett. B 711 (2012) 88 [arXiv:1108.6269] [InSPIRE].

[39] V. Bonzom, R. Gurau and M. Smerlak, Universality in p-spin glasses with correlated disorder, J. Stat. Mech. (2013) L02003 [arXiv:1206.5539].

[40] R. Gurau, The 1/N Expansion of Tensor Models Beyond Perturbation Theory, Commun. Math. Phys. 330 (2014) 973 [arXiv:1304.2666] [InSPIRE].

[41] T. Krajewski, Group field theories, PoS (QGQGS 2011) 005 [arXiv:1210.6257] [INSPIRE].

[42] S. Carrozza, Tensorial methods and renormalization in Group Field Theories, arXiv: 1310.3736

[43] D. Oriti, Disappearance and emergence of space and time in quantum gravity, Stud. Hist. Philos. Mod. Phys. 46 (2014) 186 [arXiv:1302.2849] [InSPIRE].

[44] W. Kaminski, D. Oriti and J.P. Ryan, Towards a double-scaling limit for tensor models: probing sub-dominant orders, New J. Phys. 16 (2014) 063048 [arXiv:1304.6934] [INSPIRE].

[45] V.A. Nguyen, S. Dartois and B. Eynard, An analysis of the intermediate field theory of $T^{4}$ tensor model, JHEP 01 (2015) 013 [arXiv:1409.5751] [INSPIRE]. 